\title{
Clinicopathological spectrum of uterine leiomyomas in a state of Northern India: a hospital based study
}

\author{
Mega Lahori $^{1}{ }^{*}$, Aneeta Singh Malhotra ${ }^{1}$, Sakul $^{2}$, Arvind Khajuria ${ }^{1}$, KC Goswami $^{1}$ \\ ${ }^{1}$ Department of Pathology, ${ }^{2}$ Department of Medicine, Acharya Shri Chander College of Medical Sciences, Jammu and
} Kashmir, India

Received: 14 May 2016

Accepted: 06 June 2016

\section{*Correspondence:}

Dr. Mega Lahori,

E-mail: iammegha00@gmail.com

Copyright: $\odot$ the author(s), publisher and licensee Medip Academy. This is an open-access article distributed under the terms of the Creative Commons Attribution Non-Commercial License, which permits unrestricted non-commercial use, distribution, and reproduction in any medium, provided the original work is properly cited.

\section{ABSTRACT}

Background: Myometrial lesions form a diverse group amongst which leiomyoma is the commonest visceral neoplasm affecting females in the reproductive age group. They are noted clinically in $20-30 \%$ of women over 30 years of age; are rare prior to the menarche, common in reproductive life and have a tendency to regress after the menopause. Their gross appearances are often altered by various secondary changes. Subtypes of leiomyoma are chiefly of interest as they may mimic malignancy in some cases.

Methods: All the hysterectomy and myomectomy specimens which were received in the department of pathology, ASCOMS hospital Jammu, Jammu and Kashmir over a period of one year, out of which 79 cases with leiomyomas were included in the study. The specimens were properly labeled, fixed (in NBF), examined grossly, processed, stained and examined microscopically.

Results: Age range of the patients with leiomyoma was18-62 years. Majority of the patients were between 41-50 years $(46.84 \%$ cases). Menorrhagia was the commonest symptom constituting $37.97 \%$ cases and fibroid uterus was the most common clinical diagnosis provided (44\%). Most common location of leiomyoma's was intramural $(57.43 \%)$ followed by subserosal (30.69\%). 56.96\% leiomyoma's were single and $43.04 \%$ were multiple. Degenerative changes were observed in $16.46 \%$ cases, amongst which hyaline change was the most common (6.33\%). 9 types of leiomyoma variants were seen, amongst which cellular leiomyoma (6.33\%) was the commonest. Adenomyosis was associated with leiomyoma in $19.23 \%$ cases.

Conclusions: This study was conducted to analyze the clinic pathologic spectrum of uterine leiomyoma's in northern India with regards to their clinical presentation, associated changes and variants, and to compare our findings with similar studies from different parts of the world.

Keywords: Leiomyoma, Myometrium, Hysterectomy, Myomectomy

\section{INTRODUCTION}

Myometrium (Latin myo-muscle + Greek metra-womb) is the thick, smooth muscle coat of the uterus which encases the endometrium and is lined by the peritoneum derived serosa. ${ }^{1}$ Myometrial lesions form a diverse group amongst which leiomyoma (benign smooth muscle tumor) is the commonest; followed by adenomyosis, leiomyosarcoma, endometrial stromal tumors, secondary tumors, vascular lesions, etc.
Leiomyoma is the commonest visceral neoplasm affecting females in reproductive age group. ${ }^{2}$ Fibroid, fibromyoma and myoma are the terms synonymously used with this benign neoplasm. Originally believed to be fibromas, the misnomer "fibroid" has come to be so widely employed that it is generally considered synonymous with leiomyoma. They are noted clinically in 20-30\% of women over 30 years of age, and are found in as many as $75 \%$ of uteri when a systematic search is conducted. ${ }^{3}$ They are rare prior to the menarche, common in reproductive life, have a tendency to regress after the 
menopause and are associated with endometrial hyperplasia, all of which suggest their estrogen dependence. Also, the myometrium of leiomyoma's expresses higher levels of estrogen receptors. ${ }^{4}$ The importance of leiomyoma's lies in the symptoms they cause-pain, abnormal uterine bleeding and a sensation of pressure. Large tumors produce diffuse uterine enlargement or an irregular uterine contour, which may be associated with infertility. Some leiomyoma's are pedunculated and protrude through the cervical os. ${ }^{4}$

Grossly, they are well-circumscribed, firm, gray-white bulging masses (varying in size from barely visible nodules to large tumors that fill the pelvis) that can be easily shelled out from the myometrium and have a whorled appearance on cut surface with cells arranged in crisscrossing fascicles on microscopy. They are difficult to diagnose on curettage material, since they resemble superficial myometrium. The gross appearances are often altered by secondary or degenerative changes, which are commonly seen. ${ }^{5,6}$ Hyaline degeneration/necrosis is present in more than $60 \%$, particularly in postmenopausal women, and cystic degeneration, myxoid change, fatty degeneration and calcification each occur in about $4 \%$. After menopause or delivery, leiomyomas can undergo atrophy with significant shrinkage and fibrosis. Red degeneration is associated with pregnancy and contraceptive use, and is due to tumor vessel thrombosis. $^{4,5}$

Most subtypes of leiomyoma are chiefly of interest in that they mimic malignancy in one or more respects. ${ }^{4}$ These subtypes are mitotically active leiomyoma, cellular leiomyoma, haemorrhagic cellular leiomyoma, leiomyoma with bizarre nuclei, epithelioid leiomyoma, and myxoid leiomyoma. Smooth muscle proliferations with unusual growth patterns may be in the form of diffuse leiomyomatosis, dissecting leiomyoma, parasitic leiomyoma, disseminated peritoneal leiomyomatosis, intravascular leiomyoma and benign metastasizing leiomyoma. $^{7-9}$

Only a few studies have elaborated on the clinicpathological changes seen in uterine leiomyoma's, hence in this context the present study was taken up. This is also the first comprehensive study on uterine leiomyoma pathology from Northern India.

\section{METHODS}

The material consists of all the hysterectomy and myomectomy specimens who were received in the department of pathology, Acharya Shri Chander College of medical sciences hospital Jammu, Jammu and Kashmir over a period of 1 November 2014 to 31 October 2015. A total of 165 specimens were received for histopathological examination in the department of pathology, out of which 79 cases diagnosed with leiomyoma's were included in the study. The clinical information and the relevant investigations of the patients were obtained from the histopathological requisition forms and clinical record files. The specimens received in the department of pathology were properly labeled, numbered and fixed in $10 \%$ buffered formalin. After a detailed gross examination of the specimens, multiple sections were taken from representative sites, processed and paraffin blocks were made. The blocks were sectioned and stained routinely with hematoxylin and eosin. Special stains were used wherever required.

\section{RESULTS}

Age of the patients with leiomyoma ranged from18-62 years. Majority of the patients were between $41-50$ years accounting for $46.84 \%$ cases (Table 1 ).

Table 1: Age wise distribution of patients with leiomyoma.

\begin{tabular}{|lll|}
\hline Age range (in years) & No. of cases & Percentage \\
\hline Below 20 & 01 & $1.27 \%$ \\
\hline $21-30$ & 40 & $5.06 \%$ \\
\hline $31-40$ & 16 & $20.25 \%$ \\
\hline $41-50$ & 37 & $46.84 \%$ \\
\hline $51-60$ & 19 & $24.05 \%$ \\
\hline Above 60 & 02 & $2.53 \%$ \\
\hline Total & $\mathbf{7 9}$ & $\mathbf{1 0 0 \%}$ \\
\hline
\end{tabular}

\section{Clinical presentation}

Menorrhagia was the commonest symptom constituting $37.97 \%$ cases, followed by pain in abdomen in $18.99 \%$ cases and dysmenorrhea in 17.72 cases (Table 2). Clinical diagnosis by the concerned physician was fibroid uterus in $44 \%$ cases, utero-vaginal prolapse in $20 \%$ cases, dysfunctional uterine bleeding in $19 \%$ cases and pelvic inflammatory disease in $17 \%$ cases.

Table 2: Chief complaints in patients with uterine leiomyoma.

\begin{tabular}{|lll|}
\hline Chief complaint & No. of cases & Percentage \\
\hline Menorrhagia & 30 & $37.97 \%$ \\
\hline Pain in abdomen & 15 & $18.99 \%$ \\
\hline Dysmenorrhea & 14 & $17.72 \%$ \\
\hline Mass per vaginum & 13 & $16.46 \%$ \\
\hline Post-menopausal bleeding & 04 & $5.06 \%$ \\
\hline Leucorrhoea & 02 & $2.53 \%$ \\
\hline Infertility & 01 & $1.27 \%$ \\
\hline Total & $\mathbf{7 9}$ & $\mathbf{1 0 0 \%}$ \\
\hline
\end{tabular}

Most common site of leiomyomas was intramural $(57.43 \%)$ followed by subserosal leiomyomas $(30.69 \%)$, submucosal leiomyomas constituted $8.91 \%$ cases while broad ligament leiomyomas constituted $2.97 \%$ cases.

In the present study, out of 79 cases of leiomyomas, $45(56.96 \%)$ were single and $34(43.04 \%)$ were multiple 
(Table 3). Number of leiomyomas observed in the present study varied from 1 to 10 .

Table 3: Location and number of leiomyoma's in uterus.

\begin{tabular}{|lllll|}
\hline $\begin{array}{l}\text { Location of } \\
\text { leiomyoma }\end{array}$ & $\begin{array}{l}\text { Single } \\
\text { location }\end{array}$ & $\begin{array}{l}\text { Multiple } \\
\text { location }\end{array}$ & $\begin{array}{l}\text { Total } \\
\text { number }\end{array}$ & Percentage \\
\hline Intramural & 36 & 22 & 58 & $56.86 \%$ \\
\hline Subserosal & 21 & 11 & 32 & $31.37 \%$ \\
\hline Submucous & 06 & 03 & 09 & $08.83 \%$ \\
\hline $\begin{array}{l}\text { Broad } \\
\text { ligament }\end{array}$ & 03 & 0 & 03 & $02.94 \%$ \\
\hline Total & $\mathbf{6 6}$ & $\mathbf{3 6}$ & $\mathbf{1 0 2}$ & $\mathbf{1 0 0 \%}$ \\
\hline
\end{tabular}

\section{Size of leiomyomas}

Sub-serosal leiomyomas varied from few mm to $6 \times 5 \times 4$ $\mathrm{cm}$ in size. Intramural leiomyomas varied from few $\mathrm{mm}$ to $12 \times 10 \times 8$ in diameter. Sub-mucosal leiomyomas varied from few $\mathrm{mm}$ to $3.5 \mathrm{~cm}$ in diameter.

In this study, majority of leiomyomas were diagnosed in multiparous women. Out of 79 patients with leiomyomas, $78(98.73 \%)$ were parous, which includes 10 cases of uniparous patients and only 1 was nulliparous (1.28\%).

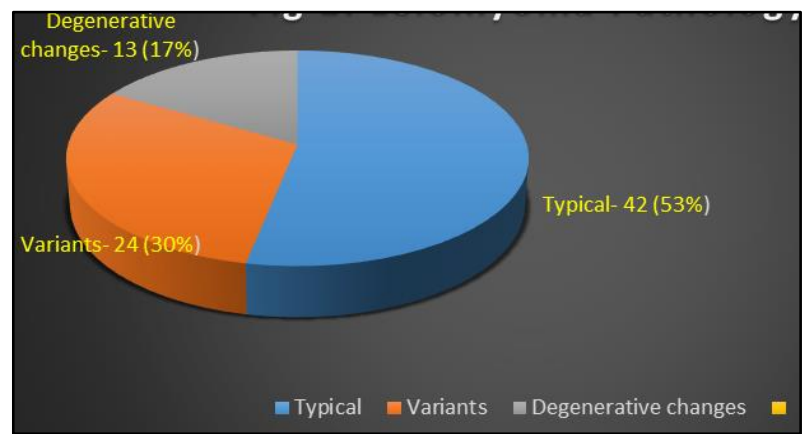

Figure 1: Various pathological changes seen in uterine leiomyomas.

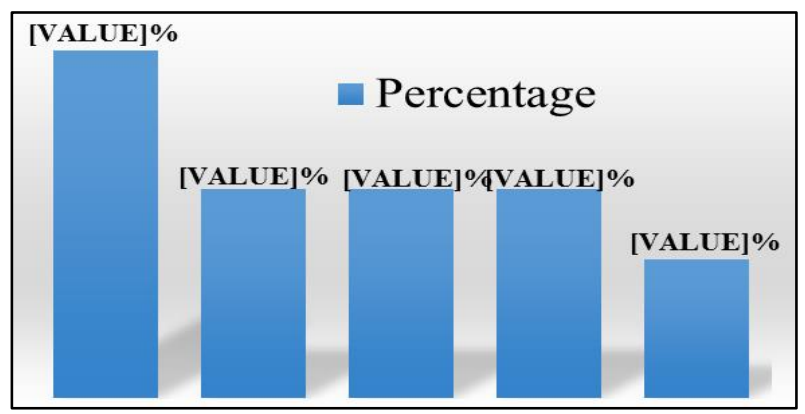

Figure 2: Various degenerative changes seen in uterine leiomyomas.

Types of leiomyomas: in our study, we observed 42 cases of typical leiomyomas $(53.16 \%)$, followed by leiomyoma variants in 24 cases $(30.38 \%)$ and degenerative changes in 13 cases $(16.46 \%)$ (Figure 1).
Degenerative changes were observed in 13 leiomyomas (16.46\%) (Figure 2). Among these, 05 leiomyomas $(6.33 \%)$ showed hyaline change which constituted the most common degenerative change observed in this study, 03 leiomyomas (3.8\%) showed myxoid change, 03 cases $(3.8 \%)$ showed calcification, 03 cases $(3.8 \%)$ showed cystic and 02 cases $(2.53 \%)$ demonstrated carneous (red) degeneration [Figure 3(c)].

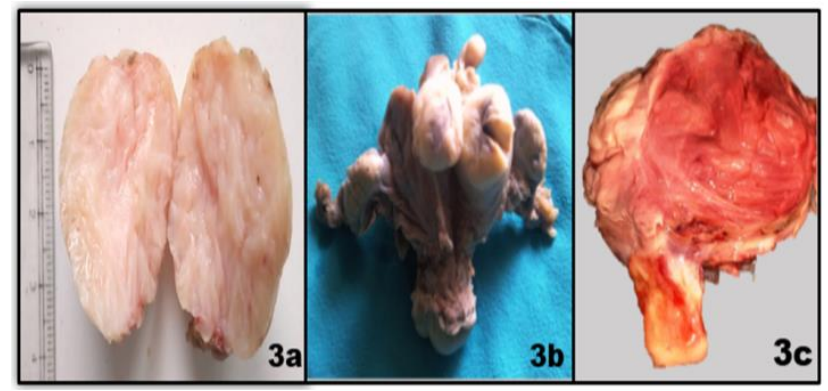

Figure 3(a): Cut section of a myomectomy specimen of whorled, raw-silk appearance typical of a fibroid; (b) gross section of uterus of diffuse leiomyomatosis; (c) cut section of uterine leiomyoma of red degeneration.

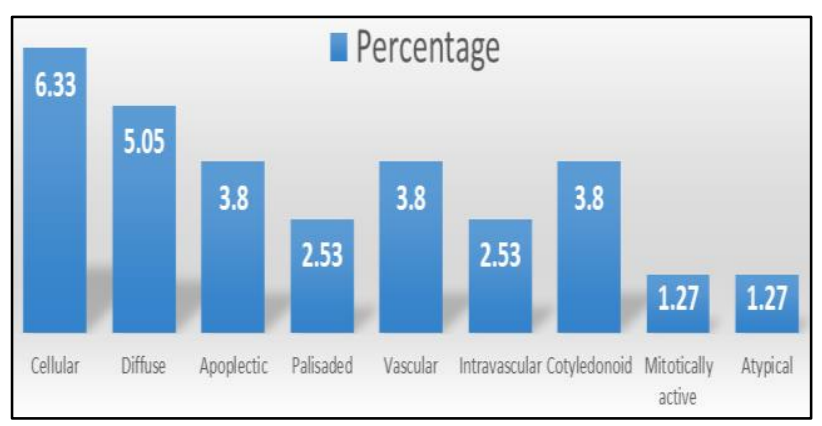

Figure 4: Variants of uterine leiomyoma's observed in the present study.

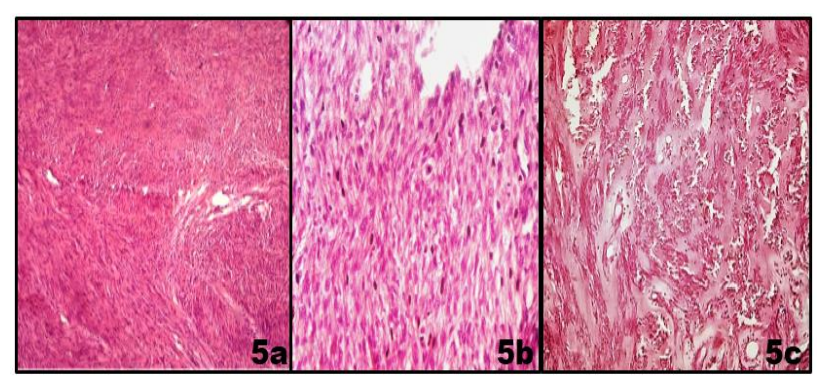

Figure 5(a): Photomicrograph of a cellular leiomyoma of dense crisscrossing trabeculae; (b)

photomicrograph of mitotically active leiomyoma demonstrating increased mitotic activity in absence of atypia; (c) Photomicrograph of a palisaded (neurilemmoma-like) leiomyoma.

Variants of leiomyoma: we observed 09 types of variants of leiomyoma in the present study of the total 79 leiomyomas (Figure 4), which included following types of variants- cellular leiomyoma (6.33\%), diffuse 
leiomyomatosis $(5.05 \%)$, apoplectic leiomyoma (3.8\%), Cotyledonoid leiomyoma $(3.8 \%)$, palisaded leiomyoma (2.53\%), vascular leiomyoma (3.8\%), intravascular leiomyoma $(2.53 \%)$, mitotically active leiomyoma $(1.27 \%)$ and atypical leiomyoma (1.27\%) (Figure 5).

Associated myometrial pathology in leiomyomas: adenomyosis was found associated with leiomyoma in 15 cases $(19.23 \%)$.

\section{DISCUSSION}

Leiomyomas continue to be a major cause of morbidity in perimenopausal women. Limited data is available from our community regarding clinicopathologic patterns of uterine leiomyomas. This study was conducted to analyze the clinicopathologic spectrum of uterine leiomyomas with regards to their presentation, location, associated changes and variants, and to compare our findings with those of other similar studies from different parts of the world.

The ages of the patients ranged from 18-62 years. The average age of patients was 45.82 years. Highest numbers of patients included in this study were between 41-50 years $(46.82 \%)$. These findings were similar to that observed by Gupta et al (51.40\%), Rather et al (47.27\%), Vaidya et al $(45.63 \%)$ and Rizvi et al $(44.56 \%) .{ }^{10-13}$ In other studies, 31-40 years age group was mainly affectedKarthikeyan et al (46.15\%), Gowri et al (41.3\%). ${ }^{14,15}$

In this study, menorrhagia was the commonest presenting symptom seen in $37.97 \%$ cases, followed by dysmenorrhea in $18.99 \%$ cases. Menorrhagia was also the presenting complaint in studies by Sarfraz (68\%), Karthikeyan (62.5\%), Rather (35.43\%), Gowri (49.03\%) and Manjula K $(35.4 \%){ }^{11,14-17}$

The most common preoperative diagnosis was fibroid uterus in $44 \%$ cases followed by utero-vaginal prolapse in $20 \%$ cases, dysfunctional uterine bleeding in $19 \%$ cases and pelvic inflammatory disease in $17 \%$ cases. These findings are consistent with the data reported by Vaidya et al $(42.96 \%$ and $18.95 \%)$, Siwatch et al $(39 \%$ and $22.6 \%$ ), utero-vaginal prolapse was the commonest indication in a study by Jha et al $(37.1 \%)$, Gupta et al $(40.0 \%) .^{10,12,18,19}$

In the present study, out of 79 cases of leiomyomas, 45 (56.96\%) were single and $34(43.04 \%)$ were multiple. In a study by Sarfraz et al (2010) multiple leiomyomas were seen in $60.87 \%$ cases. $^{16}$ Abraham and Saldanha 20 observed solitary leiomyoma in $42.5 \%$ cases and multiple leiomyomas in $57.5 \%$.

The most common site of leiomyomas in our study was intramural $(57.43 \%)$ followed by subserosal leiomyomas $(30.69 \%)$, sub mucosal leiomyomas $(8.91 \%)$ and broad ligament leiomyomas $(2.97 \%)$. Jung et al observed intramural fibroids in $55.7 \%$ cases, subserous fibroids in
$16.3 \%$ cases, $15.6 \%$, and submucosal fibroids in $12.4 \%$ cases respectively. ${ }^{21}$ Intramural leiomyomas were also the commonest types in studies by Gowri et al $(48 \%)$ and Rosario et al (52\%). ${ }^{15,22}$ Abraham and Saldanha observed intramural fibroids in $61.5 \%$ cases, subserosal leiomyomas in $9 \%$ cases and submucosal leiomyomas in $5 \%$ cases. $^{20}$

In the present study, degenerative changes were observed in 13 leiomyomas (16.46\%). Among these, $6.33 \%$ showed hyaline change which constituted the most common degenerative change observed in this study, $6.33 \%$ showed myxoid change, $3.8 \%$ showed calcification, $3.8 \%$ showed cystic and $2.53 \%$ demonstrated red (carneous) degeneration. Jung at al found secondary (degenerative) changes in $9.2 \%$ cases and the most common change was hyaline degeneration $(5.7 \%){ }^{21}$ Gowri et al reported secondary changes in $22.6 \%$ cases with hyalinization $(16.9 \%)$ being the commonest secondary degenerative change followed by cystic $(3.5 \%)$ and myxoid $(1.6 \%)$ change. ${ }^{15}$ Abraham and Saldanha observed secondary changes $22.2 \%$ cases; among these $49 \%$ showed hyaline change, $4.9 \%$ showed myxoid change, $4.9 \%$ showed calcification, 3.35 showed red degeneration and $4.9 \%$ showed hydropic change. ${ }^{20}$

In the present study, 09 variants of leiomyoma were seen in 24 cases out $(30.38 \%)$ of the total 79 leiomyomas, which included following types of variants-cellular leiomyoma (6.33\%), apoplectic leiomyoma (3.8\%), diffuse leiomyomatosis $(5.05 \%)$, cotyledonoid leiomyoma $(3.8 \%)$, palisaded leiomyoma $(2.53 \%)$, vascular leiomyoma $(3.8 \%)$, intravascular leiomyoma $(2.53 \%)$, mitotically active leiomyoma (1.27\%) and atypical leiomyoma (1.27\%). Abraham and Saldanha in their study encountered leiomyoma variants in $7.5 \%$ cases, which of which $78 \%$ were cellular leiomyomas, $9.5 \%$ were lipoleiomyoma and $4.7 \%$ were bizarre (symplastic) leiomyomas and $2.3 \%$ were epithelioid leiomyomas. ${ }^{20}$ Manjula $\mathrm{K}$ et al in their study observed leiomyoma variants in $4.55 \%$ cases, which includedLipoleiomyoma $(2.05 \%)$, myxoid $(0.91 \%)$, hemorrhagic cellular $(0.45 \%)$, cellular $(0.22 \%)$, epithelioid $(0.22 \%)$, bizarre $(0.22 \%)$, palisaded $(0.22 \%)$ and lymphocytic infiltrated $(0.22 \%)$ variants. $^{17}$

\section{CONCLUSION}

Leiomyoma is the most common benign tumor of the pelvis. They are commonly seen in perimenopausal females and present with menorrhagia, pain in abdomen or dysmenorrhea. Intramural site was the most common location, hyaline change was the most common degeneration and cellular variant was the most common subtype seen in our study. The treatment modalities are chiefly hysterectomy, myomectomy or drug therapy (GNRH analogues), depending on various factors like parity, size, symptomatology, etc. Also, the pathologist needs to be cautious while diagnosing cases of atypical, mitotically active or bizarre leiomyoma's due to their 
morphologic homogeneity with STUMP and leiomyosarcoma.

\section{ACKNOWLEGEMENT}

Authors would like to thanks Mr Rajesh Verma and $\mathrm{Mr}$ Vinod for the technical help provided.

Funding: No funding sources

Conflict of interest: None declared

Ethical approval: Not Required

\section{REFERENCES}

1. Christopher PC. The female genital tract in: Kumar, Abbas, Fauster eds. Robbins and Cottron Pathologic basis of disease. $8^{\text {th }}$ Ed. India Elsevier; 2010:1036-8.

2. Silverberg SG, Tabbara SO. The uterine corpus. In: Silverberg SG, Delellis RA, Frable WJ, Eds. Principles and Practice of Surgical Pathology and Cytopathology. Vol 3 ( $3^{\text {rd }}$ edition). New York: Churchill Livingstone; 1997:2459-516.

3. Cramer SF, Patel A. The frequency of uterine leiomyomas. Am J Clin Pathol. 1990;94:435-8.

4. Zaloudek CJ, Hendrickson MR, Soslow RA. Mesenchymal tumors of uterus. In: Blaustein Pathology of the female genital tract. $6^{\text {th }}$ ed; 2011:459-466.

5. Persaud V, Arjoon PD. Uterine leiomyoma: incidence of degenerative change and a correlation of associated symptoms. Obstetrics and Gynaecology. 1970;35(3):329-492.

6. Samaila Modupeola OA, Adesiyun AG, Agunbiade OA, Mohammed D. A clinicopathological assessment of hysterectomies in Zaria. Eur $\mathbf{J}$ Gen Med. 2009;6(3):150-3.

7. Ojeda VJ. The pathology of hysterectomy specimens. NZ Med J. 1979;89(631):169-71.

8. Adelusola KA, Ogunniyi SO. Hysterectomies in Nigerians: histopathological analysis of cases seen in Ile-Ife. Niger Postgrad Med Journal. 2001;8:37-40.

9. Smooth JS, Zaloudek C. Myometrial and stromal lesions of the uterus Gynaecologic pathology. Clinics in Lab Medicine. 1995;15(3):545-73.

10. Gupta G, Kotasthane D, Kotasthane V. Hysterectomy: a clinico-pathological correlation of
500 cases. The Internet Journal of Gynecology and Obstetrics. 2009;14(1).

11. Rather GM, Gupta Y, Bardhwaj S. Patterns of lesions in hysterectomy specimens: a prospective study. JK Science. 2013;15(2):35-8.

12. Vaidya S, Vaidya SA. Patterns of lesions in hysterectomy specimens in a tertiary care hospital. J Nepal Med Assoc. 2015;53(197):18-23.

13. Rizvi G, Pandey H, Pant H, Chufal SS, Pant P. Histopathological correlation of adenomyosis and leiomyoma in hysterectomy specimens as the cause of abnormal uterine bleeding in women in different age groups in the Kumaon region: a retro prospective study. J Midlife Health. 2013;4(1):27-30.

14. Karthikeyan TM, Veenaa NN, Ajeeth Kumar CR, Thomas E. Clinico-pathological study of hysterectomy among rural patients in a tertiary care center. IOSR Journal of Dental and Medical Sciences. 2015;14(5):25-7.

15. Gowri M, Mala G, Murthy S, Nayak V. Clinicopathological study of uterine leiomyomas in hysterectomy specimens. Journal of Evolution of Medical and Dental Sciences. 2013;2(46):9002-9.

16. Sarfraz R, Sarfraz MA, Kamal F, Afsar A. Pattern of benign morphological myometrial lesions in total abdominal hysterectomy specimens. Biomedica. 2010;26:140-3.

17. Manjula K, Rao KS, Chandrasekhar HR. Variants of Leiomyoma: histomorphological study of tumors of myometrium. Journal of South Asian Federation of Obstetrics and Gynecology. 2011;3(2):89-92.

18. Siwatch S, Kundu R, Mohan H, Huria A. Histopathologic audit of hysterectomy specimens in a tertiary care hospital. Sri Lanka J Obstet Gynaecol. 2012;34(4):155-8.

19. Jha R, Pant AD, Jha A, Adhikari RC, Sayami G. Histopathological analysis of hysterectomy specimens. J Nep Med Assoc. 2006;45:283-90.

20. Abraham J, Saldanha P. Morphological variants and secondary changes in uterine leiomyomas. Is it important to recognize them? Int $\mathbf{J}$ of Biomed Reseach. 2013;4(12):254-64.

21. Jung JK, Koi MS, Jung BW, Lee HH, Choi HJ, Shin SK. A clinical analysis of uterine myoma. Korean $\mathbf{J}$ Obstet Gynecol. 1998;41(1):210-9.

22. Rosario YP. Uterine fibromyomas. J of Obstet and Gynaecol of India. 1968;18:101-7.

Cite this article as: Lahori M, Malhotra AS,

Sakul, Khajuria A, Goswami KC.

Clinicopathological spectrum of uterine

leiomyomas in a state of Northern India: a hospital

based study. Int J Reprod Contracept Obstet

Gynecol 2016;5:2295-9. 